\title{
Distributed Adaptive Sliding Mode Control for Vehicle Platoon with Uncertain Driving Resistance and Actuator Saturation
}

\author{
Jia-cheng Song $\mathbb{D}$ and Yong-feng Ju $\mathbb{i}$ \\ School of Electronic and Control Engineering, Chang'an University, Xi'an 710064, China \\ Correspondence should be addressed to Jia-cheng Song; songjiacehng@hotmail.com and Yong-feng Ju; yfju@chd.edu.cn
}

Received 9 January 2020; Revised 1 June 2020; Accepted 16 June 2020; Published 13 July 2020

Guest Editor: Karthikeyan Rajagopal

Copyright (C) 2020 Jia-cheng Song and Yong-feng Ju. This is an open access article distributed under the Creative Commons Attribution License, which permits unrestricted use, distribution, and reproduction in any medium, provided the original work is properly cited.

\begin{abstract}
Vehicle platoon has been demonstrated to be a promising driving pattern for its prominent advantages in enhancing traffic safety, improving highway capacity, and increasing fuel economy as well as reducing carbon emissions. However, the uncertain driving resistance and saturated actuator output decay the control performance and may even lead to the instability of a vehicle platoon. Therefore, a distributed adaptive sliding mode control algorithm for vehicle platoon with uncertain driving resistance and actuator saturation is proposed in this paper. First of all, sliding mode control technique, together with the coupled sliding surface (CSS) method, is adopted to design the vehicle platoon control algorithm and an adaptive updating law is proposed to estimate the unknown driving resistance coefficients. Then, for the problem of actuator saturation, an antiwindup compensation-based approach is utilized to attenuate the integral windup of the adaptive platoon control laws in the case of actuator saturation. In addition, considering the chattering problem inherent in sliding mode control, a sigmoid-like function sgn $(\cdot)$ is deployed to weaken the influence of chattering, which is expected to enhance the driving comfortableness. Both theoretical analysis and numerical simulation verify the feasibility and effectiveness of the proposed vehicle platoon algorithm.
\end{abstract}

\section{Introduction}

In recent years, the automated highway system (AHS) has gained considerable attentions from governments, automobile manufactures, and academia because of the increasing traffic congestion problem in large cities [1-3]. As an effective measure to relieve congestions, platoon control $[4,5]$, which requires vehicles to move in a string with predefined intervehicle distance and with the same velocity, has demonstrated its unique advantage in enhancing traffic safety, improving highway capacity, increasing fuel economy, and reducing carbon emissions [6-8].

During the past few years, many achievements have been developed for vehicle platoon control, such as backstepping approach [9], information consensus approach [10, 11], adaptive sliding mode approach [12], etc. However, most of the above works treat the vehicle platoon control problem in a simplified fashion without considering the driving resistance inherent in the vehicle dynamics. As a matter of fact, driving resistance, which includes rolling resistance, air resistance, and grade resistance [13], has significant influence on vehicle platoon such as degrading the controller performance and leading to the instability of a vehicle string [14]. In addition, driving resistance is virtually influenced by many factors such as vehicle mass, motorcycle type, and road and weather conditions $[15,16]$, some of which may even vary with the driving conditions [17]. Therefore, it is a great challenge in obtaining the driving resistance parameters in an accurate way.

For the problem of unknown driving resistance in vehicle dynamics, Altmannshofer and Endisch [18] proposed a robust parameter estimation algorithm for identifying the vehicle driving resistance. Tannoury et al. [16] designed a nonlinear observer for the estimation of tire radius and 
rolling resistance to compensate for the unknown parameters. Guo et al. [14] deployed a quadratic function to represent the unknown and time-varying coefficients of driving resistance. However, as for the unknown driving resistance in vehicle platoon control, existing literatures mainly treat it as time-varying external disturbance without explicitly obtaining its true value [12]. In [9], the uncertain driving resistance in vehicle dynamics is modeled as an unknown time-varying disturbance and an adaptive method is adopted to estimate this value. Similarly, the uncertain driving resistance is described as a bounded disturbance and also estimated by adaptive approach in [14]. In [13], the vehicle resistance, which is relevant to vehicle mass, weather conditions, deadweight, motorcycle type, etc., is analyzed and the vehicle platoon model is established.

On the other hand, the mechanical constraints, especially the actuator saturation [19], are important concern in vehicle dynamics. The actuator saturation of vehicle, whether it is the servo motor for an electric vehicle or engine for a gasoline vehicle, has been proved to be the source of performance degradation in vehicle driving procedure [20], which therefore deserves further investigation in vehicle platoon control. However, to the best of our knowledge, literatures that specifically address this issue seem very few. As a ubiquitous phenomenon in mechanical systems, actuator saturation, which is usually referred to as input saturation, has been intensively investigated in various control domains. In [20], by explicitly considering the actuator saturation, a novel robust adaptive control law is proposed to ensure the stability of the closed-loop system for high-speed train. In [21], the Nussbaum function is introduced to compensate for the nonlinear term arising from input saturation. In [22], a saturated adaptive robust control strategy, by adding an anti-windup block, is designed for vehicle active suspension systems, which is beneficial for the stability and performance preservation in the presence of saturation. In [23], an adaptive coordinated control algorithm of multiple high-speed trains with input saturation is proposed, where an antiwindup compensation block is used to optimize the control algorithm such that it is more resilient to input saturation. In [24], a smooth function $\tanh (\cdot)$ is introduced to handle the "actuator saturation" problem in vehicle platoon such that the control input can always be below the maximum inputs. Therefore, the above work provides fruitful inspirations for the actuator saturation problem of vehicle platoon.

In this paper, we are trying to investigate the vehicleplatoon problem with uncertain driving resistance and actuator saturation via adaptive sliding mode control approach. Firstly, coupled sliding surface (CSS) is deployed to link interconnected vehicles and an adaptive control method is adopted to identify and estimate the variations of resistance coefficients. After that, a distributed adaptive sliding mode control algorithm for vehicle platoon with uncertain driving resistance is proposed. Then, an antiwindup compensation based approach is utilized to attenuate the integral windup of the adaptive platoon control laws in case of actuator saturation. Moreover, the chattering phenomena inherent in sliding mode control are relieved by using a sigmoid-like function. Finally, various numerical simulations are performed to demonstrate the feasibility and effectiveness of the proposed control algorithm.

The rest of this paper is organized as follows. In Section 2 , the vehicle dynamic model and the problems considered in this paper are described. In Section 3, the adaptive sliding mode controller is designed to realize the vehicle platoon in the presence of uncertain driving resistance and actuator saturation. Simulations are performed in Section 4 to demonstrate the feasibility and effectiveness of our algorithm. Concluding remarks are given in Section 5.

\section{Vehicle Dynamics and Problem Formulation}

Assume a vehicle platoon, which consists of a string of autonomous vehicles, includes a leader vehicle and $n$ followers. As is shown in Figure 1, each follower regulates its motion according to the received information (e.g., position, velocity, and acceleration) from its front and back vehicles via wireless communication technique.

2.1. Vehicle Dynamics. Consider a vehicle platoon moving in a string with the following longitudinal dynamics:

$$
\left\{\begin{array}{l}
\dot{r}_{i}(t)=v_{i}(t), \\
M_{i} \dot{v}_{i}(t)=F_{i}(t)-f_{i}, \\
i=1,2, \ldots, n,
\end{array}\right.
$$

where $M_{i}$ is the mass of the $i$ th vehicle, $r_{i}(t)$ and $v_{i}(t)$ denote the $i$ th vehicle's position and velocity, respectively, and $F_{i}(t)$, which is taken as the control input, represents the traction or braking force of the $i$ th vehicle. In addition, $f_{i}$ denotes its driving resistance.

Generally, $f_{i}$ is influenced by the rolling resistance $f_{\mathrm{r}}$, air resistance $f_{\mathrm{w}}$, and grade resistance $f_{\mathrm{g}}$, etc. [25], which can be described as

$$
f_{i}=f_{\mathrm{r}}+f_{\mathrm{w}}+f_{\mathrm{g}}
$$
follows:

The explicit form of $f_{\mathrm{r}}, f_{\mathrm{w}}$, and $f_{\mathrm{g}}$ can be written as

(i) Rolling resistance $f_{\mathrm{r}}$ :

$$
f_{\mathrm{r}}=W k
$$

where $W$ is the normal load and $k$ is the rolling resistance coefficient following such experience values as

$$
k=0.0076+0.000056 v,
$$

where $v$ is the velocity of vehicle.

(ii) Air resistance $f_{\mathrm{w}}$ :

$$
f_{\mathrm{w}}=\frac{1}{2} C_{D} A \rho v^{2},
$$

where $C_{D}$ is the coefficient of air resistance, $A$ is windward area of vehicle, and $\rho$ is the air density. 


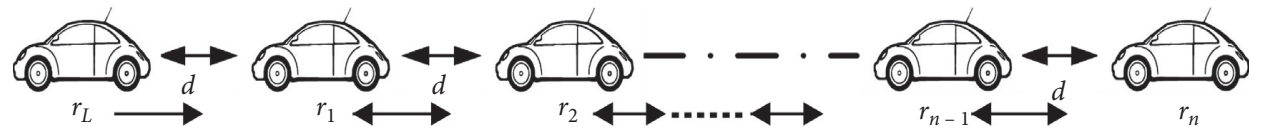

FIgURE 1: Topological structure of vehicle platoon.

(iii) Grade resistance $f_{\mathrm{g}}$ :

If the vehicle is running on the hill, the component of gravity along the slope is defined as the grade resistance

$$
f_{\mathrm{g}}=G \sin \alpha
$$

where $G$ and $\alpha$ are, respectively, the gravity and roadgrade.

As $k, C_{D}$, and $\rho$ are uncertain values and heterogeneous with respect to different vehicles, therefore, $f_{i}$ is timevarying in different driving speed and road conditions. To facilitate the research, we write $f_{i}$ as

$$
f_{i}=A(t)+B(t) v_{i}+C(t) v_{i}^{2},
$$

where $A(t), B(t), C(t)$, describing the uncertainty of driving resistance, are unknown and time-varying values.

In order to simplify the protocol design and stability analysis, we rewrite (1) as

$$
\left\{\begin{array}{l}
\dot{r}_{i}(t)=v_{i}(t) \\
\dot{v}_{i}(t)=F_{i}(t)-a_{i}(t)-b_{i}(t) v_{i}(t)-c_{i}(t) v_{i}(t)^{2}
\end{array}\right.
$$

where $u_{i}(t):=\left(F_{i}(t) / M_{i}\right)$ denotes the acceleration or deceleration of vehicle $i$ (it is designed as the control input in this paper) and $a_{i}(t):=\left(A_{i}(t) / M_{i}\right), b_{i}(t):=\left(B_{i}(t) / M_{i}\right)$, $c_{i}(t):=\left(C_{i}(t) / M_{i}\right)$ are the vehicle driving resistance coefficients by considering the influence of vehicle mass $M_{i}$.

2.2. Problem Formulation. Generally speaking, the main purpose of vehicle platoon is to enhance the highway capacity and relieve traffic congestion by maintaining the desired safety distance between two consecutive vehicles and reaching the velocity consensus among vehicles [4].

In particular, the time-varying driving resistance will inevitably influence the vehicle dynamics and decay the vehicle platoon performance. Therefore, one needs to specifically design the distributed control input $u_{i}(t)$ for a single vehicle such that the vehicle platoon is achieved under the disturbance of time-varying driving resistance.

In addition, due to the physical and mechanical limitations of actuators, the control input $u_{i}(t)$ for vehicles will be under some constraints. The control input $u_{i}(t)$ with saturation is given in the following form:

$$
\begin{aligned}
u_{i}(t) & =\operatorname{sat}\left(u_{\min }, u_{i}(t), u_{\max }\right) \\
& = \begin{cases}u_{\min }, & \text { if } u_{i}(t)<u_{\min }, \\
u_{i}(t), & \text { if } u_{\min } \leq u_{i}(t) \leq u_{\max } \\
u_{\max }, & \text { if } u_{i}(t)>u_{\max },\end{cases}
\end{aligned}
$$

where $u_{\min }$ and $u_{\max }$ are known constants, which represent the bounds of the control force.

Based on above description, the main control objective of this paper can be concluded as follows:

(1) The vehicle platoon is achieved such that the follower's velocity can converge to the velocity of the leader and each vehicle can maintain a safe intervehicle distance to avoid collision with each other

(2) The unknown time-varying coefficients $a_{i}(t), b_{i}(t)$, and $c_{i}(t)$ can be estimated such that the vehicle platoon is achieved by handling the parameter uncertainties via an adaptive control approach

(3) When the control input exceeds the maximum output of the vehicle actuator (servo motor for an electric vehicle or engine for a gasoline vehicle), the proposed algorithm can regulate the actuator output autonomously such that the actuator life as well as the vehicle platoon performance is guaranteed

\section{Distributed Adaptive Sliding Mode Control Algorithm for Vehicle Platoon}

Firstly, the position tracking error for the $i$ th vehicle is defined as

$$
e_{i}=\left(r_{i-1}-r_{i}\right)-d,
$$

where $d>0$ is a constant value, representing the required distance between two consecutive vehicles.

We also denote the velocity error by

$$
\widetilde{v}_{i}=v_{i-1}-v_{i} \text {. }
$$

Here, the following assumptions are made for facilitating the control protocol design and theoretical analysis.

Assumption 1. $a_{i}(t), b_{i}(t)$, and $c_{i}(t)$ are bounded variables; i.e., $a_{i}(t) \leq a_{i}^{+}, b_{i}(t) \leq b_{i}^{+}$, and $c_{i}(t) \leq c_{i}^{+}$.

3.1. Vehicle Platoon Control Algorithm with Uncertain Driving Resistance. We first consider the case of vehicle platoon with uncertain driving resistance. For the dynamics of vehicles with error $e_{i}$, the control objective is to make $e_{i}$ converge to zero and to guarantee string stability.

Hence, sliding mode control technique is employed to develop the vehicle platoon controller; we choose each sliding surface as

$$
n_{i}=\dot{e}_{i}+\alpha_{i} e_{i},
$$

where $\alpha_{i}>0$ is a positive constant. 
The solution of (12) is

$e_{i}(t)=\frac{n_{i}}{\alpha_{i}}+\exp \left(-\alpha_{i} t\right) e_{i}(0)-\frac{n_{i}}{\alpha_{i}} \exp \left(-\alpha_{i}\left(t-t_{0}\right)\right)$.

From (13), one can see that when $t \longrightarrow \infty$, $e_{i} \longrightarrow\left(n_{i} / \alpha_{i}\right)$. As $\alpha_{i}$ is a positive constant, $e_{i} \Leftrightarrow n_{i}$. Furthermore, combined with (12), we have $\dot{e}_{i} \longrightarrow 0$ when $n_{i} \longrightarrow 0$.

Taking the time derivative of (12), one obtains

$$
\dot{n}_{i}=\ddot{e}_{i}+\alpha_{i} \dot{e}_{i}=\left(\ddot{r}_{i-1}-\ddot{r}_{i}\right)+\alpha_{i} \dot{e}_{i} \text {. }
$$

It is worth noting that (14) only describes the characteristic of a single vehicle. In order to describe the stability of the whole platoon, we adopt the coupled sliding surface (CSS) [7] of the $i$ th vehicle for the control of the whole platoon system

$$
N_{i}=\beta_{i} n_{i}-n_{i+1}
$$

where $\beta_{i}>0(i=1,2, \ldots, n)$ is a weighting factor.

Since $n_{i+1}$ does not exist for the last vehicle (i.e., $i=n$ ), we set $n_{i+1}=0$. Then, we have $\mathbf{N}_{1}:=\left[n_{1}, n_{2}, \ldots, n_{n}\right]^{T}$ and $\mathbf{N}_{2}$ : $=\left[N_{1}, N_{2}, \ldots, N_{n}\right]^{T}$; the relationship between $\mathbf{N}_{1}$ and $\mathbf{N}_{2}$ can be described as

$$
\mathbf{N}_{2}=\mathbf{B N}_{1},
$$

where

$$
\mathbf{B}=\left[\begin{array}{ccccc}
\beta_{1} & -1 & 0 & \cdots & 0 \\
0 & \beta_{2} & -1 & \cdots & 0 \\
\vdots & \vdots & \vdots & \ddots & \vdots \\
0 & 0 & 0 & \cdots & -1 \\
0 & 0 & 0 & \cdots & \beta_{n}
\end{array}\right],
$$

with $\beta_{i}>0(i=1,2, \ldots, n)$ being the parameters to be designed.

In order to illustrate the same convergence of $\mathbf{N}_{1}$ and $\mathbf{N}_{2}$, the following lemma is given.

Lemma 1 (see [7]). Equivalence of the convergence of the CSS and each sliding surface toward zero: $\mathbf{N}_{2}$ becomes zero if and only if $\mathbf{N}_{1}$ becomes zero at the same time.

Therefore, the problem of making $n_{i}$ and $e_{i}$ converge to zero is converted into making $N_{i}$ converge to zero. The time derivative of $N_{i}$ in (15) can be written as

$$
\begin{aligned}
\dot{N}_{i} & =\beta_{i} \dot{n}_{i}-\dot{n}_{i+1} \\
& =\beta_{i}\left(\ddot{r}_{i-1}-\ddot{r}_{i}+\alpha_{i} \dot{e}_{i}\right)-\left(\ddot{r}_{i}-\ddot{r}_{i+1}+\alpha_{i+1} \ddot{e}_{i+1}\right) \\
& =-\left(\beta_{i}+1\right)\left(u_{i}-a_{i}-b_{i} v_{i}-c_{i} v_{i}^{2}\right)+D_{i},
\end{aligned}
$$

where $D_{i}=\beta_{i} \ddot{r}_{i-1}+\ddot{r}_{i+1}+\alpha_{i} \beta_{i} \dot{e}_{i}-\alpha_{i+1} \dot{e}_{i+1}$.

Accordingly, the novel adaptive platoon control law for the $i$ th vehicle is designed as

$$
u_{i}=\frac{k_{1}}{\beta_{i}+1} N_{i}+\frac{1}{\beta_{i}+1} D_{i}+\operatorname{sgn}\left(N_{i}\right)\left(\widehat{a}_{i}^{+}+\widehat{b}_{i}^{+} v_{i}+\widehat{c}_{i}^{+} v_{i}^{2}\right),
$$

where $k_{1}$ is a positive parameter that needs to be designed and $\widehat{a}_{i}^{+}, \widehat{b}_{i}^{+}$, and $\widehat{c}_{i}^{+}$are the estimated values of unknown constant coefficients $a_{i}^{+}, b_{i}^{+}$, and $c_{i}^{+}$.

The adaptive estimation law for unknown coefficients is determined by

$$
\left\{\begin{array}{l}
\dot{\hat{a}}_{i}^{+}=\lambda_{1}\left(\beta_{i}+1\right) N_{i}, \\
\dot{\hat{b}}_{i}^{+}=\lambda_{2}\left(\beta_{i}+1\right) N_{i}\left|v_{i}\right|, \\
\dot{\hat{c}}_{i}^{+}=\lambda_{3}\left(\beta_{i}+1\right) N_{i} v_{i}^{2},
\end{array}\right.
$$

where $\lambda_{1}, \lambda_{2}, \lambda_{3}>0$ are positive constants.

Particularly, when $i=n$, we know $N_{n}=\beta_{n} n_{n}$ from the definition of (15); the time derivative of $N_{n}$ can be described as

$$
\begin{aligned}
\dot{N}_{n} & =\beta_{n} \dot{n}_{n} \\
& =\beta_{n}\left(\ddot{r}_{n-1}-\ddot{r}_{n}+\alpha_{n} \dot{e}_{n}\right) \\
& =-\beta_{n}\left(u_{n}-a_{n}-b_{n} v_{n}-c_{n} v_{n}^{2}\right)+D_{n},
\end{aligned}
$$

where $D_{n}=\beta_{n}\left(\ddot{r}_{n-1}+\alpha_{n} \dot{e}_{n}\right)$ for $i=n$.

The adaptive platoon control law for the $n$th vehicle is therefore formulated as

$$
u_{n}=\frac{k_{2}}{\beta_{n}} N_{n}+\frac{1}{\beta_{n}} D_{n}+\operatorname{sgn}\left(N_{n}\right)\left(\widehat{a}_{n}^{+}+\widehat{b}_{n}^{+} v_{n}+\widehat{c}_{n}^{+} v_{n}^{2}\right),
$$

where $k_{2}$ is a positive parameter that needs to be designed.

Thus, the coefficients adaptation law can be designed as

$$
\left\{\begin{array}{l}
\dot{\hat{a}}_{n}^{+}=\lambda_{1} \beta_{n} N_{n}, \\
\dot{\vec{b}}_{n}^{+}=\lambda_{2} \beta_{n} N_{n}\left|v_{n}\right|, \\
\dot{\hat{c}}_{n}^{+}=\lambda_{3} \beta_{n} N_{n} v_{n}^{2} .
\end{array}\right.
$$

Then, the following theorem, which guarantees the stability of each vehicle and string stability of the whole vehicle platoon, can be obtained.

Theorem 1. Consider a vehicle platoon described by (8), the proposed control algorithm of vehicle platoons (19) and (22) and the adaptive control law of coefficients (20) and (23) can ensure that the sliding surfaces $N_{i}$ and $n_{i}$ and the distance error $e_{i}$ converge to zero.

Proof. First, we define the estimation error of coefficients $\widetilde{a}_{i}^{+}$, $\widetilde{b}_{i}^{+}$, and $\widetilde{c}_{i}^{+}$as

$$
\left\{\begin{array}{l}
\widetilde{a}_{i}^{+}=\widehat{a}_{i}^{+}-a_{i}^{+}, \\
\tilde{b}_{i}^{+}=\widehat{b}_{i}^{+}-b_{i}^{+}, \\
\widetilde{c}_{i}^{+}=\widehat{c}_{i}^{+}-c_{i}^{+} .
\end{array}\right.
$$

Choose the following Lyapunov function candidates:

$$
V=\sum_{i=1}^{n} V_{i}
$$

where

$$
V_{i}=\frac{1}{2} N_{i}^{2}+\frac{1}{2 \lambda_{1}} \widetilde{a}_{i}^{+} 2+\frac{1}{2 \lambda_{2}} \widetilde{b}_{i}^{+} 2+\frac{1}{2 \lambda_{3}} \widetilde{c}_{i}^{+} 2 .
$$


Then, the time derivative of $V_{i}$ can be calculated as

$$
\dot{V}_{i}=N_{i} \dot{N}_{i}+\frac{1}{\lambda_{1}} \widetilde{a}_{i}^{+} \dot{\vec{a}}_{i}^{+}+\frac{1}{\lambda_{2}} \widetilde{b}_{i}^{+} \dot{\vec{b}}_{i}^{+}+\frac{1}{\lambda_{3}} \widetilde{c}_{i} \dot{\tilde{c}}_{i}^{+} .
$$

$\dot{\tilde{b}}_{i}^{+}$Beçause $a_{i}^{+}, b_{i}^{+}$, and $c_{i}^{+}$are constants, we know $\dot{\tilde{a}}_{i}^{+}=\dot{\hat{a}}_{i}^{+}$, $\dot{\vec{b}}_{i}^{+}=\widehat{b}_{i}$, and $\dot{\vec{c}}_{i}^{+}=\dot{\hat{c}}_{i}^{+}$according to (24). Combining (18) and (20) with (27) yields

$$
\begin{aligned}
\dot{V}_{i}= & -k_{1} N_{i}^{2}-\left(\beta_{i}+1\right) N_{i}\left[\left(\widehat{a}_{i}^{+}-a_{i}\right)+\left(\hat{b}_{i}^{+}-b_{i}\right) v_{i}+\left(\widehat{c}_{i}^{+}-c_{i}\right) v_{i}^{2}\right] \\
& +\left(\beta_{i}+1\right) N_{i}\left(\widetilde{a}_{i}^{+}+\widetilde{b}_{i}^{+} v_{i}+\widetilde{c}_{i}^{+} v_{i}^{2}\right) .
\end{aligned}
$$

According to Young's inequality [14], we have

$$
\left\{\begin{array}{l}
a_{i} \leq a_{i}^{+} \\
b_{i} v_{i} \leq b_{i}^{+}\left|v_{i}\right| \\
c_{i} v_{i}^{2} \leq c_{i}^{+} v_{i}^{2}
\end{array}\right.
$$

Substituting (29) into (28) gives

$$
\begin{aligned}
\dot{V}_{i} \leq & -k_{1} N_{i}^{2}-\left(\beta_{i}+1\right) N_{i}\left[\left(\widehat{a}_{i}^{+}-a_{i}^{+}\right)+\left(\widehat{b}_{i}^{+}-b_{i}^{+}\right) v_{i}+\left(\widehat{c}_{i}^{+}-c_{i}^{+}\right) v_{i}^{2}\right] \\
& +\left(\beta_{i}+1\right) N_{i}\left(\widetilde{a}_{i}^{+}+\widetilde{b}_{i}^{+} v_{i}+\widetilde{c}_{i}^{+} v_{i}^{2}\right) \\
\leq & -k_{1} N_{i}^{2} .
\end{aligned}
$$

Thus,

$$
\dot{V} \leq-k_{1} \sum_{i=1}^{n} N_{i}^{2} \leq 0 .
$$

From (30) and (31), we know that $\dot{V}_{i}$ and $\dot{V}$ are negative semidefinite; therefore, $V_{i}$ and $V$ are not monotonic increasing and boundedness. In addition, we can also obtain that $N_{i}$ is boundedness and $\dot{N}_{i}$ is boundedness.

Taking the derivative of (30) and (31), we have $\ddot{V}_{i} \leq-\left(k_{1} / 2\right) N_{i} \dot{N}_{i}, \ddot{V} \leq\left(k_{1} / 2\right) \sum_{i=1}^{n} N_{i} \dot{N}_{i}$. As $\ddot{V}_{i}$ and $\ddot{V}$ are boundedness, $\dot{V}_{i}$ and $\dot{V}$ are uniformly continuous. According to the Barbalat lemma, we know that $\lim _{t \longrightarrow \infty} V_{i}=0$ and $\lim _{t \rightarrow \infty} \dot{V}=0$. Since $k_{1}$ is positive, $\lim _{t \rightarrow \infty} N_{i}=0$ for all $i$. This implies that $\mathbf{N}_{2}, \mathbf{N}_{1}$, and $e_{i}$ in Lemma 1 converge to zero.

Remark 1. Cconstrued sliding surface (12) facilitates the control design and stability analysis. Open-loop dynamics (12) contains $e_{i}$ and $\dot{e}_{i}$; the closed-loop dynamics $\dot{n}_{i}$ will contain system dynamics (8) and the control input $u_{i}$. Inspired by the adaptive technique, the control inputs (19) and (22) and the adaptive laws (19) and (23) can be designed by choosing the suitable Lyapunov function.

Remark 2. The main objective of the designed controller is to make $N_{i}, \widetilde{a}_{i}^{+}, \widetilde{b}_{i}^{+}$, and $\widetilde{c}_{i}^{+}$converge to zero; thus the Lyapunov function $V_{i}$ is chosen as (26). The principle of controller design is to make $\dot{V}_{i} \leq 0$.

\subsection{Vehicle Platoon Control Algorithm with Uncertain Driving} Resistance and Actuator Saturation. In practice, the actuator saturation of vehicles has proved to be a source of performance degradation [26]. To handle this problem, an antiwindup compensation block is used to modify the control input such that it is more resilient to actuator saturation. An antiwindup compensator is used to generate a signal $\phi_{i}(t)$ for each vehicle as the output of a differential equation:

$$
\left\{\begin{array}{l}
\dot{\phi}_{i}(t)=-\chi_{i} \phi_{i}(t)+\left(\beta_{i}+1\right)\left(u_{i 0}(t)-u_{i}(t)\right), \\
\dot{\phi}_{n}(t)=-\chi_{n} \phi_{n}(t)+\beta_{n}\left(u_{n 0}(t)-u_{n}(t)\right) .
\end{array}\right.
$$

Let $\delta_{i}(t)=N_{i}(t)-\phi_{i}(t)$. We have the following error dynamic system:

$$
\left\{\begin{array}{l}
\dot{\delta}_{i}(t)=-\left(\beta_{i}+1\right)\left(u_{i}(t)-a_{i}-b_{i} v_{i}-c_{i} v_{i}^{2}\right)-\left(\beta_{i}+1\right)\left(u_{i 0}(t)-u_{i}(t)\right)+D_{i}+\chi_{i} \phi_{i}(t), \\
\dot{\delta}_{n}(t)=-\beta_{n}\left(u_{n}(t)-a_{n}-b_{n} v_{n}-c_{n} v_{n}^{2}\right)-\beta_{n}\left(u_{n 0}(t)-u_{n}(t)\right)+D_{n}+\chi_{n} \phi_{n}(t) .
\end{array}\right.
$$

By considering explicitly the actuator saturation of vehicles, the modified adaptive control input $u_{i}(t)$ for each vehicle can be written as

$$
\begin{gathered}
u_{i}(t)=\operatorname{sat}\left(u_{\min }, u_{i 0}(t), u_{\max }\right), \\
u_{i 0}(t)=\frac{k_{1}}{\beta_{i}+1} \delta_{i}+\frac{1}{\beta_{i}+1} D_{i}-\frac{1}{\beta_{i}+1} \chi_{i} \phi_{i}(t) \\
+\operatorname{sgn}\left(\delta_{i}\right)\left(\widehat{a}_{i}^{+}+\hat{b}_{i}^{+} v_{i}+\widehat{c}_{i}^{+} v_{i}^{2}\right) .
\end{gathered}
$$

The adaptive estimation laws for unknown coefficients are determined by

$$
\left\{\begin{array}{l}
\dot{\vec{a}}_{i}^{+}=\lambda_{1}\left(\beta_{i}+1\right) \delta_{i}, \\
\dot{\widehat{b}}_{i}^{+}=\lambda_{2}\left(\beta_{i}+1\right) \delta_{i}\left|v_{i}\right|, \\
\dot{\widehat{c}}_{i}^{+}=\lambda_{3}\left(\beta_{i}+1\right) \delta_{i} v_{i}^{2} .
\end{array}\right.
$$

The adaptive platoon control law of the $n$th vehicle is therefore formulated as

$$
u_{n 0}(t)=\frac{k_{n}}{\beta_{n}} \delta_{n}+\frac{1}{\beta_{n}} D_{n}-\frac{1}{\beta_{n}} \chi_{n} \phi_{n}(t)+\operatorname{sgn}\left(\delta_{n}\right)\left(\widehat{a}_{n}^{+}+\widehat{b}_{n}^{+} v_{n}+\widehat{c}_{n}^{+} v_{n}^{2}\right) .
$$

The coefficients adaptation law is designed as 


$$
\left\{\begin{array}{l}
\dot{\hat{a}}_{n}^{+}=\lambda_{1} \beta_{n} \delta_{n}, \\
\dot{\hat{b}}_{n}^{+}=\lambda_{2} \beta_{n} \delta_{n}\left|v_{n}\right|, \\
\dot{\hat{c}}_{n}^{+}=\lambda_{3} \beta_{n} \delta_{n} v_{n}^{2} .
\end{array}\right.
$$

The signal $\phi_{i}(t)$ is used for attenuating the integral windup of the adaptive coordinated control laws in case of actuator saturation. When actuator saturation happens, there will be a rise of error $\phi_{i}(t)$, and meanwhile the signal $N_{i}$ will also increase, which in turn ensures that no sudden rise will happen to the newly defined tracking error $\delta_{i}(t)$. When the actuator saturation stops, the term $u_{i 0}(t)-u_{i}(t)=0$, and the signal $\phi_{i}(t)$ will converge to zero.

In addition, for the signal $\phi_{i}(t)$, we have the following theorem.

Theorem 2. For the signal $\phi_{i}(t)$, there exists a positive $\omega_{i}$ such that, for any $t>0$, it holds that

$$
\left|\phi_{i}(t)\right| \leq\left|\frac{\left(u_{i 0}(t)-u_{i}(t)\right)\left(\beta_{i}+1\right)}{\chi_{i}}\right|+\varpi_{i} .
$$

Proof. From (32), we can obtain that

$$
\begin{aligned}
\phi_{i}(t) & =\phi_{i}(0) e^{-\chi_{i} t}+\frac{\left(u_{i 0}(t)-u_{i}(t)\right)\left(\beta_{i}+1\right)}{\chi_{i}} e^{-\chi_{i} t} \int_{0}^{t} e^{-\chi_{i} \varpi} \mathrm{d} \omega \\
& =\phi_{i}(0) e^{-\chi_{i} t}+\frac{\left(u_{i 0}(t)-u_{i}(t)\right)\left(\beta_{i}+1\right)}{\chi_{i}}\left(1-e^{-\chi_{i} t}\right),
\end{aligned}
$$

which implies that $\left|\phi_{i}(t)\right| \leq\left|\left(u_{i 0}(t)-u_{i}(t)\right)\left(\beta_{i}+1\right) / \chi_{i}\right|$ as $t \longrightarrow \infty$. Thus, there exists a positive scaler $\aleph_{i}$, such that, for any $t>0$, it holds that $\left|\phi_{i}(t)\right| \leq\left|\left(u_{i 0}(t)-u_{i}(t)\right)\left(\beta_{i}+1\right) / \chi_{i}\right|$; i.e., $\phi_{i}(t)$ is bounded.

The following theorem will provide our result on the vehicle platoon with actuator saturation.

Theorem 3. Consider a vehicle platoon described by (8), the proposed control algorithm of vehicle platoon (35) and (37) and the adaptive control law of coefficients (36) and (38) can ensure the desired distances between two consecutive vehicles. Then, we have the following results:

(1) When the saturation does not occur, i.e., $u_{i}-u_{i 0}=0$, all the results in Theorems 1 and 2 will hold automatically.

(2) When the saturation occurs, i.e., $u_{i}-u_{i 0} \neq 0$, the modified $\delta_{i}(t)$ will converge to zero, and $N_{i}$ will also converge to zero by adjusting variable $\chi_{i}$ in Theorem 2. This implies that $\mathbf{N}_{2}, \mathbf{N}_{1}$, and $e_{i}$ in Lemma 1 converge to zero.
Proof. For the case that the actuator is not saturated, $u_{i}-u_{i 0}=0$, the results in Theorem 1 hold automatically.

When saturation occurs, for the error dynamic (33) of the vehicles movement with control input (35) and (37), construct the following Lyapunov-like function candidate:

$$
V=\sum_{i=1}^{n} V_{i}
$$

where

$$
V_{i}=\frac{1}{2} \delta_{i}^{2}+\frac{1}{2 \lambda_{1}} \tilde{a}_{i}^{+} 2+\frac{1}{2 \lambda_{2}} \widetilde{b}_{i}^{+} 2+\frac{1}{2 \lambda_{3}} \widetilde{c}_{i}^{+} 2 .
$$

Then, the time derivative of $V_{i}$ can be calculated as

$$
\dot{V}_{i}=\delta_{i} \dot{\delta}_{i}+\frac{1}{\lambda_{1}} \tilde{a}_{i}^{+} \dot{\vec{a}}_{i}^{+}+\frac{1}{\lambda_{2}} \widetilde{b}_{i}^{+} \dot{\vec{b}}_{i}^{+}+\frac{1}{\lambda_{3}} \widetilde{c}_{i}^{+} \dot{\vec{c}}_{i}^{+} .
$$

Because $a_{i .+}^{+}, b_{i}^{+}$, and $c_{i}^{+}$are constants, we know that $\dot{\tilde{a}}_{i}^{+}=\dot{\hat{a}}_{i}^{+}, \dot{\vec{b}}_{i}^{+}=\dot{\vec{b}}_{i}^{+}$, and $\dot{\vec{c}}_{i}^{+}=\dot{\hat{c}}_{i}^{+}$according to (24). Therefore,

$$
\begin{aligned}
\dot{V}_{i} \leq & -k_{1} \delta_{i}^{2}-\left(\beta_{i}+1\right) \delta_{i}\left[\left(\hat{a}_{i}^{+}-a_{i}^{+}\right)+\left(\hat{b}_{i}^{+}-b_{i}^{+}\right) v_{i}+\left(\widehat{c}_{i}^{+}-c_{i}^{+}\right) v_{i}^{2}\right] \\
& +\left(\beta_{i}+1\right) \delta_{i}\left(\tilde{a}_{i}^{+}+\widetilde{b}_{i}^{+} v_{i}+\widetilde{c}_{i}^{+} v_{i}^{2}\right) \\
\leq & -k_{1} \delta_{i}^{2} .
\end{aligned}
$$

Thus,

$$
\dot{V} \leq-k_{1} \sum_{i=1}^{n} \delta_{i}^{2} \leq 0 .
$$

Using the same analysis method as in Theorem 1, it can be shown that $\delta_{i}$ will converge to zero eventually. From Theorem 2, we know that variable $\chi_{i}$ can be adjusted, and $\phi_{i}(t)$ can also be adjusted to an arbitrarily small value. Therefore, we have $N_{i} \approx \delta_{i}$. Combining Theorem 2 and equations (12) and (15), we know that $n_{i}$ and $e_{i}$ will converge to zero eventually.

3.3. Reduction of Chattering. It is well known that the sliding mode control has inherently the phenomena of chattering, which is detrimental for the system performance [27]. In this paper, a sigmoid-like function $\left(\delta_{i} /\left(\left|\delta_{i}+\varsigma\right|\right)\right)$ is used to replace the $\operatorname{sgn}(\cdot)$ function, where $\varsigma$ is a small positive constant.

Then, the control input $u_{i}(t)$ of the $i$ th vehicle can be rewritten as

$$
u_{i}(t)=\operatorname{sat}\left(u_{\min }, u_{i 0}(t), u_{\max }\right)
$$

where $u_{i 0}(t)$ is the calculated control input. It is determined by 
$u_{i 0}(t)=\frac{k_{1}}{\beta_{i}+1} \delta_{i}+\frac{1}{\beta_{i}+1} D_{i}-\frac{1}{\beta_{i}+1} \chi_{i} \phi_{i}(t)+\frac{\delta_{i}}{\left|\delta_{i}\right|+\varsigma}\left(\hat{a}_{i}^{+}+\hat{b}_{i}^{+} v_{i}+\hat{c}_{i}^{+} v_{i}^{2}\right)$.

The adaptive estimation law of $i$ th vehicle for unknown coefficients is determined by

$$
\left\{\begin{array}{l}
\dot{\vec{a}}_{i}^{+}=\frac{\lambda_{1}\left(\beta_{i}+1\right) \delta_{i}^{2}}{\left|\delta_{i}\right|+\varsigma}, \\
\dot{\vec{b}}_{i}^{+}=\frac{\lambda_{2}\left(\beta_{i}+1\right) \delta_{i}^{2}\left|v_{i}\right|}{\left|\delta_{i}\right|+\varsigma}, \\
\dot{\vec{c}}_{i}^{+}=\frac{\lambda_{3}\left(\beta_{i}+1\right) \delta_{i}^{2} v_{i}^{2}}{\left|\delta_{i}\right|+\varsigma} .
\end{array}\right.
$$

Similar to the previous case, $u_{n 0}(t)$ of the $n$th vehicle can be described as

$$
u_{i 0}(t)=\frac{k_{1}}{\beta_{n}} \delta_{n}+\frac{1}{\beta_{n}} D_{n}-\frac{1}{\beta_{n}} \chi_{n} \phi_{n}(t)+\frac{\delta_{n}}{\left|\delta_{n}\right|+\varsigma}\left(\widehat{a}_{n}^{+}+\hat{b}_{n}^{+} v_{n}+\widehat{c}_{n}^{+} v_{n}^{2}\right) .
$$

The adaptive estimation law of the $n$th vehicle for unknown coefficients is determined by

$$
\left\{\begin{array}{l}
\dot{\hat{a}}_{n}^{+}=\frac{\lambda_{1} \beta_{n} \delta_{n}^{2}}{\left|\delta_{n}\right|+\varsigma} \\
\dot{\vec{b}}_{n}^{+}=\frac{\lambda_{2} \beta_{n} \delta_{n}^{2}\left|v_{n}\right|}{\left|\delta_{n}\right|+\varsigma} \\
\dot{\vec{c}}_{n}^{+}=\frac{\lambda_{3} \beta_{n} \delta_{n}^{2} v_{n}^{2}}{\left|\delta_{n}\right|+\varsigma} .
\end{array}\right.
$$

\section{Numerical Simulations}

To verify the feasibility and effectiveness of the proposed platoon control algorithm, numerical simulations are performed with 8 vehicles (1 leader and 7 followers).

4.1. Simulation Setup. Without loss of generality, we suppose the vehicle platoon drives in various driving conditions, such as acceleration, cruising, and braking. In addition, we also consider the influence of time-varying driving resistance as a disturbance on the vehicle velocity in the form of sine wave.

Therefore, the desired velocity of the leading vehicle is specifically designed as

$$
\begin{cases}v_{L}(t)=20 \sin \left(\frac{\pi}{200} t\right), & \text { if } t<100, \\ v_{L}(t)=20, & \text { if } 100 \leq t<150, \\ v_{L}(t)=20+3 \sin \left(\frac{\pi}{10} t\right), & \text { if } 150 \leq t<170, \\ v_{L}(t)=20, & \text { if } 170 \leq t<230, \\ v_{L}(t)=20+3 \sin \left(\frac{\pi}{10} t\right), & \text { if } 230 \leq t<250, \\ v_{L}(t)=20, & \text { if } 250 \leq t<320, \\ v_{L}(t)=20+3 \sin \left(\frac{\pi}{10} t\right), & \text { if } 320 \leq t<340, \\ v_{L}(t)=20 \sin \left(\frac{\pi}{200} t\right), & \text { if } 400 \leq t<500, \\ v_{L}(t)=20, & \text { if } 340 \leq t<400,\end{cases}
$$

Inspired by our previous work on adaptive control for vehicle platoon [24], the variation of driving resistance coefficients $a_{i}, b_{i}, c_{i}$ is assumed to be determined by $a_{i}=a 1(1+\sin (t)), \quad b_{i}=b 1(1+\cos (3 t)), \quad c_{i}=c 1(1+\cos$ $(4 t))$, respectively. It should be pointed out that $a_{i}, b_{i}, c_{i}$ are time-varying and bounded, so they can model the uncertainty and external noise of the vehicle. The other parameters such as $a 1, b 1, c 1$, initial states of vehicles, and control parameters are listed in Tables 1 and 2 .

4.2. Simulation Results. To better illustrate the effectiveness of the proposed vehicle platoon control algorithm, three simulation cases are performed.

Case 1. Platoon with uncertain driving resistance.

In this case, we only consider the influence of uncertain driving resistance for vehicle platoon, where (19), (20), (22), and (23) are applied to the vehicle.

Figure 2(a) shows the vehicle velocities and velocity errors (between followers and leader) in the simulation, from which one can clearly see that the velocity of followers will approach the leader's velocity and the velocity errors will decline to an extremely small value around zero. Figure 2(b) gives the position curves of all vehicles in the platoon, from which we can see that vehicles will not collide during the whole driving cycle. Figure 2(c) also demonstrates that all vehicles will keep a given distance (about $10 \mathrm{~m}$ ) under the proposed platoon 
TABLE 1: Initial states of each vehicle and initial estimated values of driving resistance coefficients.

\begin{tabular}{lcccccccc}
\hline Vehicle & Leader & V1 & V2 & V3 & V4 & V5 & V6 \\
\hline$v(0)$ & 0 & 0 & 0 & 0 & 0 & 0 & 0 & 0 \\
$\dot{v}(0)$ & 0 & 0 & 0 & 0 & 0 & 0 & 0 & 0 \\
$r(0)$ & 68 & 63 & 46 & 38 & 30 & 21 & 0 \\
$\widehat{a}^{+}(0)$ & 0 & 0.8 & 0.9 & 0.85 & 0.85 & 0.8 & 0.85 & 0.8 \\
$\widehat{b}^{+}(0)$ & 0 & 0.004 & 0.004 & 0.0045 & 0.004 & 0.0045 & 0.004 & 0.0045 \\
$\hat{c}^{+}(0)$ & 0 & 0.00016 & 0.00016 & 0.00016 & 0.00016 & 0.00016 & 0.00016 & 0.00016 \\
$a 1$ & 0 & 0.3 & 0.4 & 0.4 & 0.4 & 0.5 & 0.4 & 0.5 \\
$b 1$ & 0 & 0.004 & 0.003 & 0.005 & 0.004 & 0.005 & 0.004 & 0.005 \\
$c 1$ & 0 & 0.00018 & 0.00016 & 0.00014 & 0.00017 & 0.00015 & 0.00017 & 0.00015 \\
\hline
\end{tabular}

TABLE 2: Control parameters of vehicles.

\begin{tabular}{cccccccc}
\hline$d$ & $\alpha_{i}$ & $\beta_{i}$ & $\lambda_{i}$ & $k_{1}$ & $k_{2}$ & $u_{\min }$ & $u_{\max }$ \\
\hline 10 & 100 & 5 & $10^{-10}$ & 0.5 & 0.5 & -2 & 2 \\
\hline
\end{tabular}

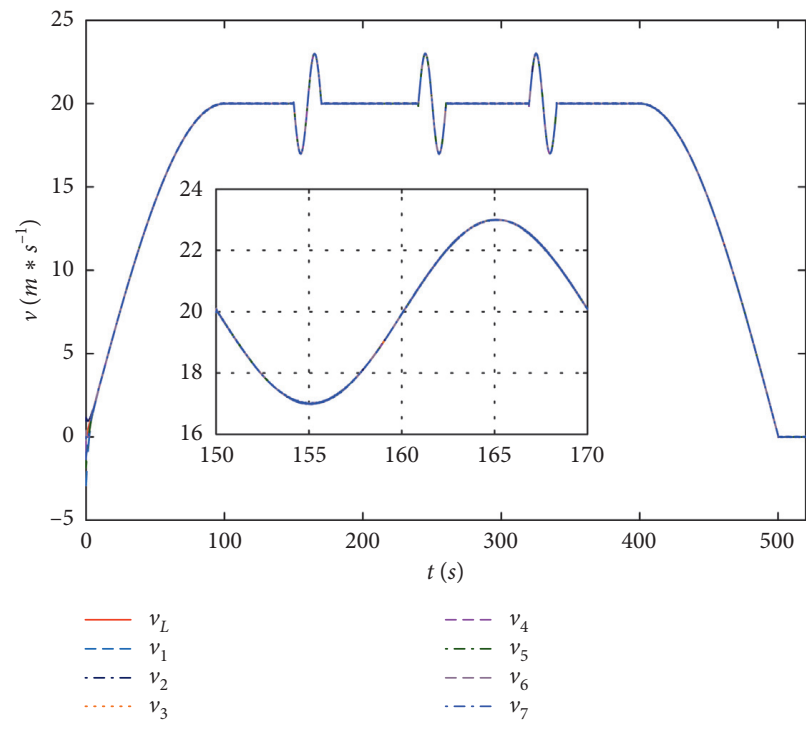

(a)

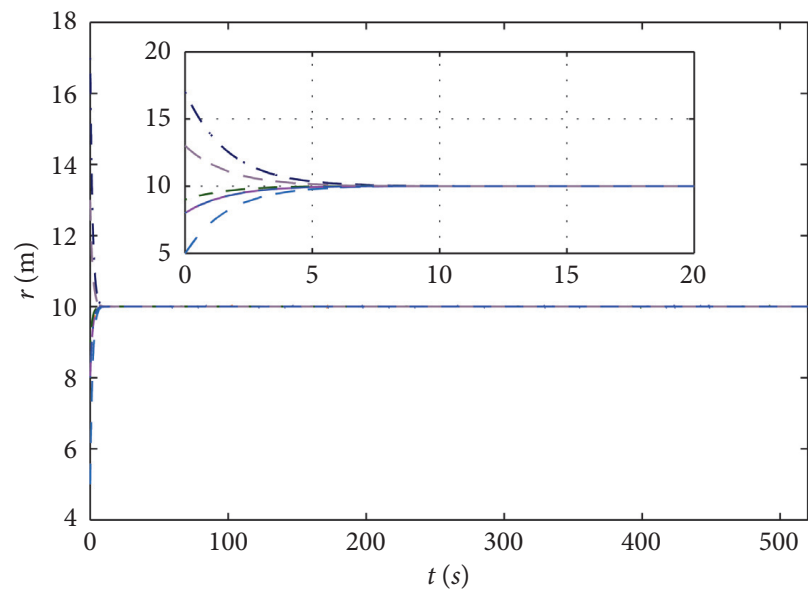

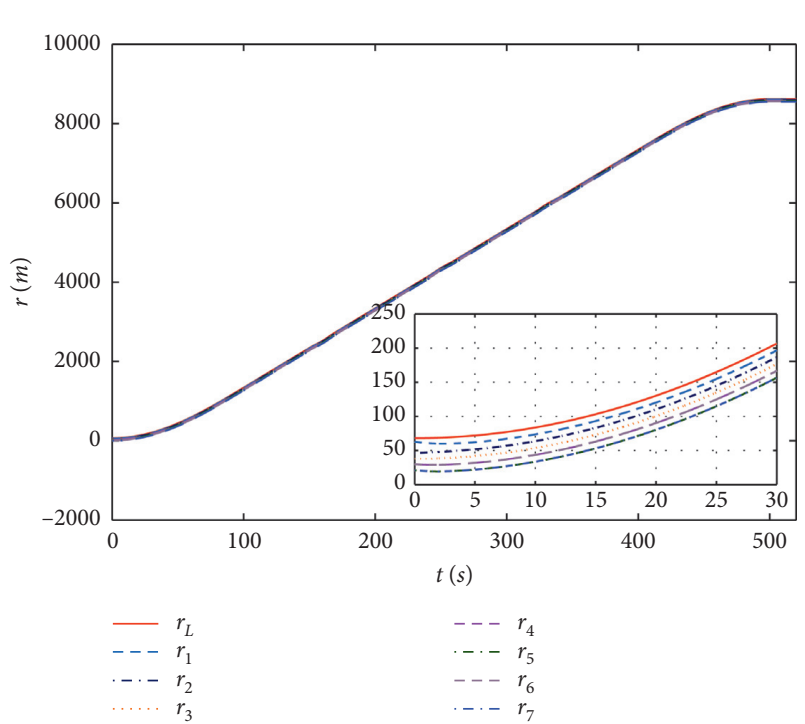

(b)

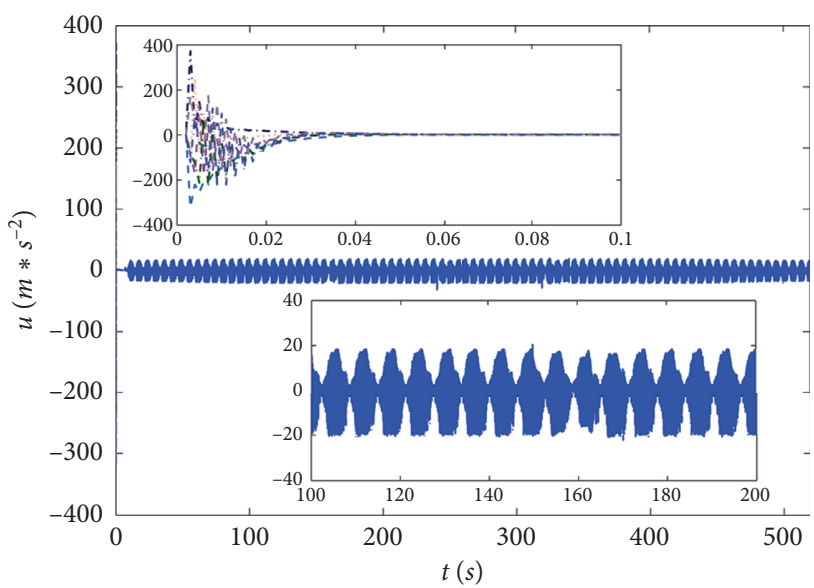

(d)

FIGURE 2: The simulation results of each vehicle with uncertain driving resistance: (a) the velocity curves; (b) the position curves; (c) the distances curves between two neighbor vehicles; (d) the control input curves. 


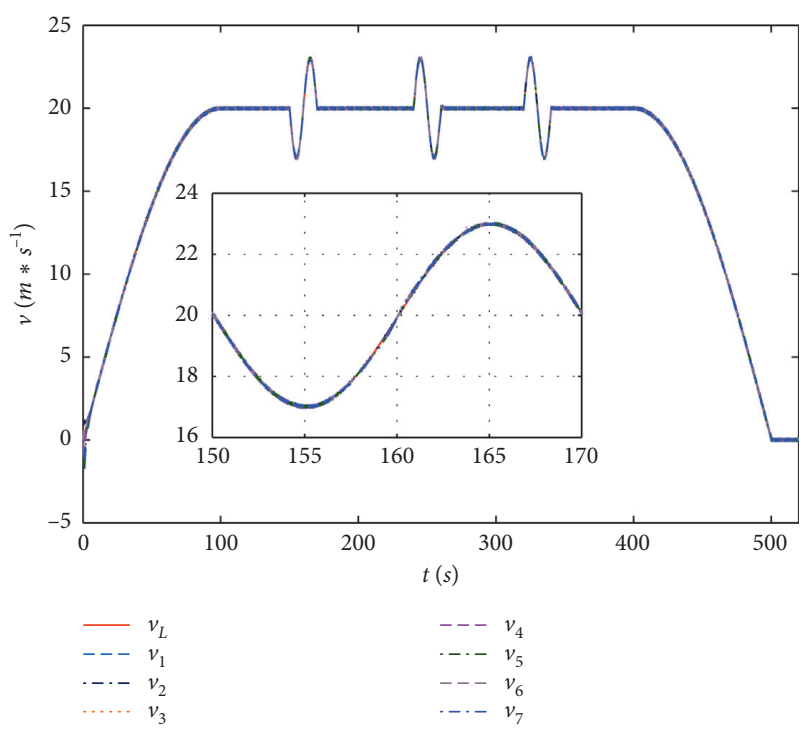

(a)

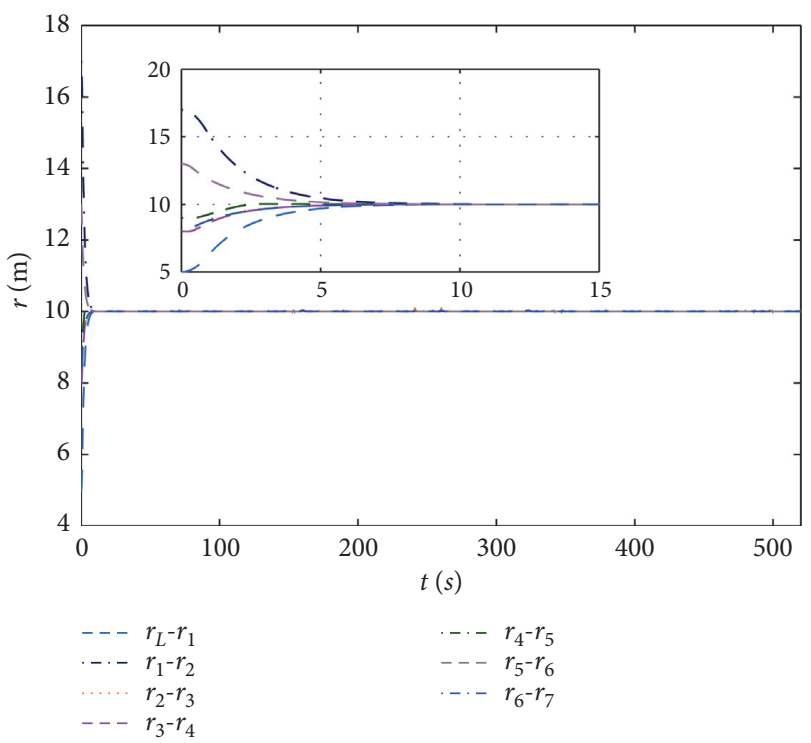

(c)

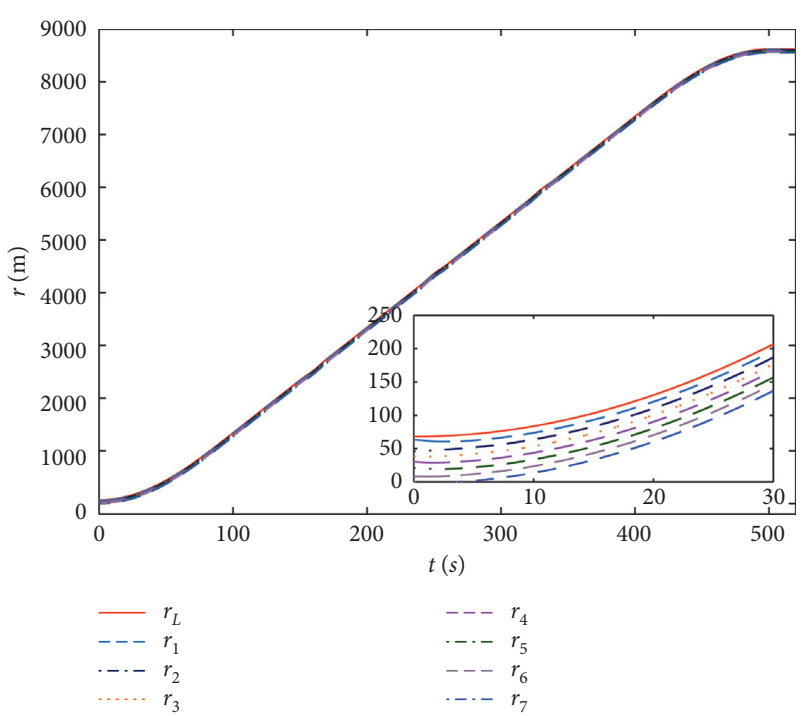

(b)

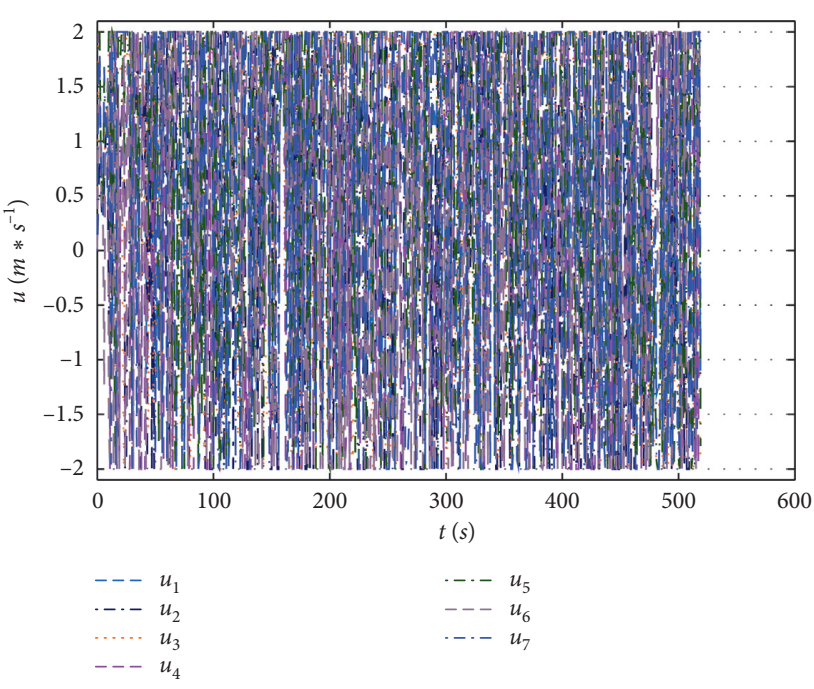

(d)

Figure 3: The simulation results of each vehicle with uncertain driving resistance and actuator saturation: (a) the velocity curves; (b) the position curves; (c) the distances curves between two neighbor vehicles; (d) the control input curves.

algorithms (19) and (22). Figure 2(d) shows the control input (acceleration or deceleration) of all followers, from which we can see that the control input decreases sharply from $400 \mathrm{~m} / \mathrm{s}^{2}$ to $20 \mathrm{~m} / \mathrm{s}^{2}$ within about $0.03 \mathrm{~s}$. Then, the control input will converge to a relatively steady state (around $20 \mathrm{~m} / \mathrm{s}^{2}$ and $-20 \mathrm{~m} / \mathrm{s}^{2}$ ). However, such control input is infeasible in practical applications, as it exceeds the maximum output of vehicle actuator and causes the phenomena of actuator saturation. Therefore, we will consider the problem of actuator saturation in Case 2.

Case 2. Platoon with actuator saturation.

In this case, the control laws (34), (35), and (37) and coefficients adaptive laws (36) and (38) are applied to the vehicle platoon. We set the initial values of signal $\phi_{i}$ as $\phi_{i}=0$ and $\dot{\phi}_{i}=0$ for $i=1,2, \ldots, n$. The parameter of $\chi_{i}$ is chosen as $\chi_{i}=100$ for $i=1,2, \ldots, n$.

Figure 3(a) shows the vehicle velocities and velocity errors (between followers and leader) by considering the influence of actuator saturation in the simulation. Compared with Figure 2(a), the control algorithms (34), (35), and (37) can also guarantee the performance of vehicle velocities and velocity errors. From Figures 3(b) and 3(c), we can also see that the proposed algorithms (34), (35), and (37) can maintain a desired intervehicle distance with their neighbors to ensure driving safety. Figure 3(d) gives the control input of all followers; it is obvious that the input (acceleration or deceleration) can be limited to $2 \mathrm{~m} / \mathrm{s}$ by adopting the proposed control algorithms (34), (35), and (37), which satisfies the maximum output of vehicle actuators. 


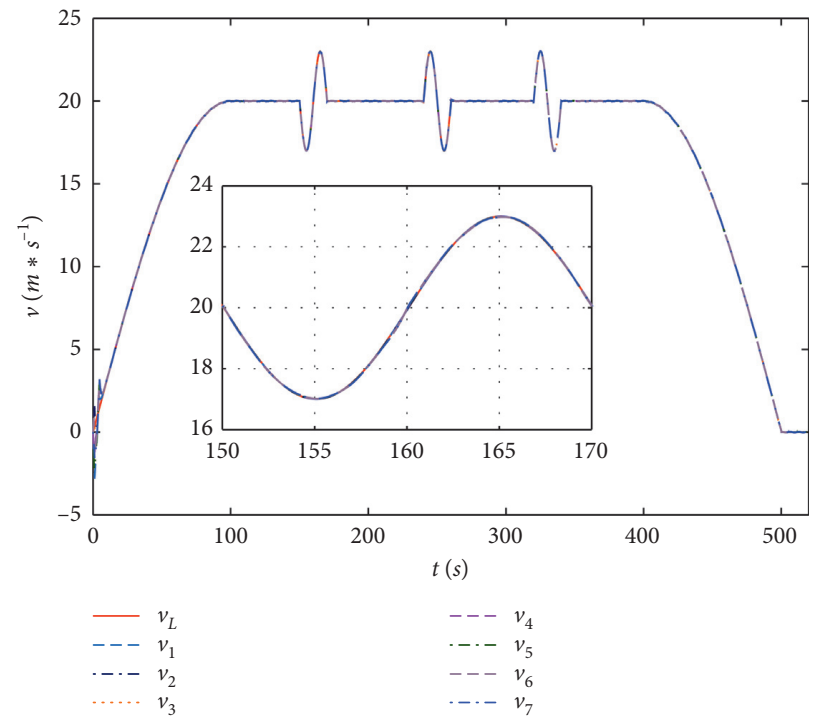

(a)

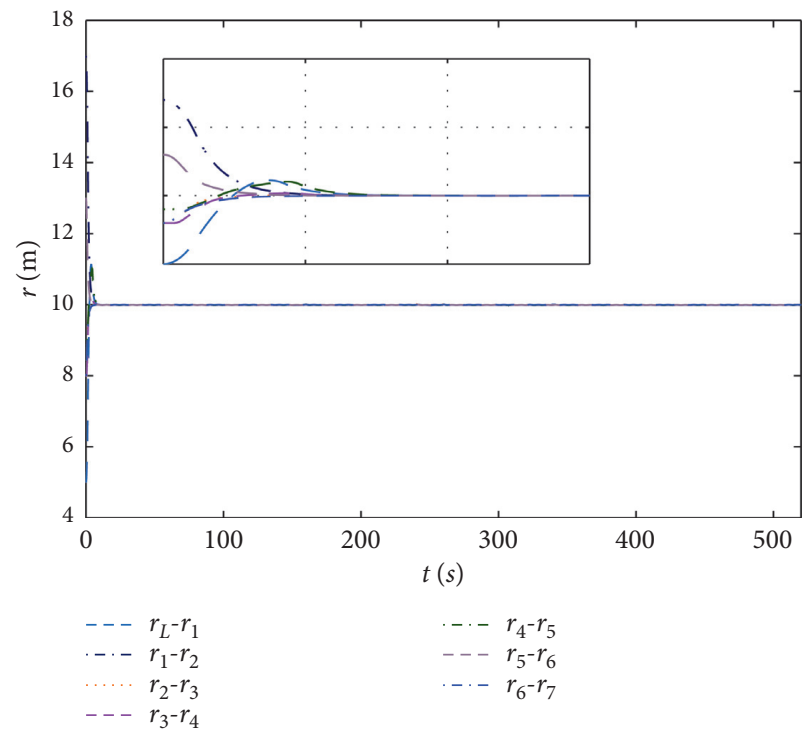

(c)

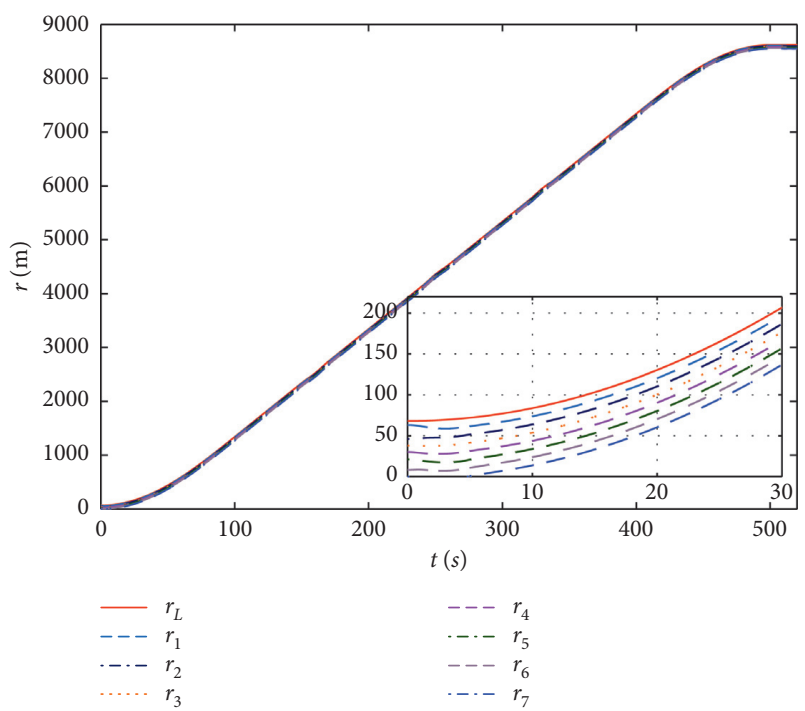

(b)

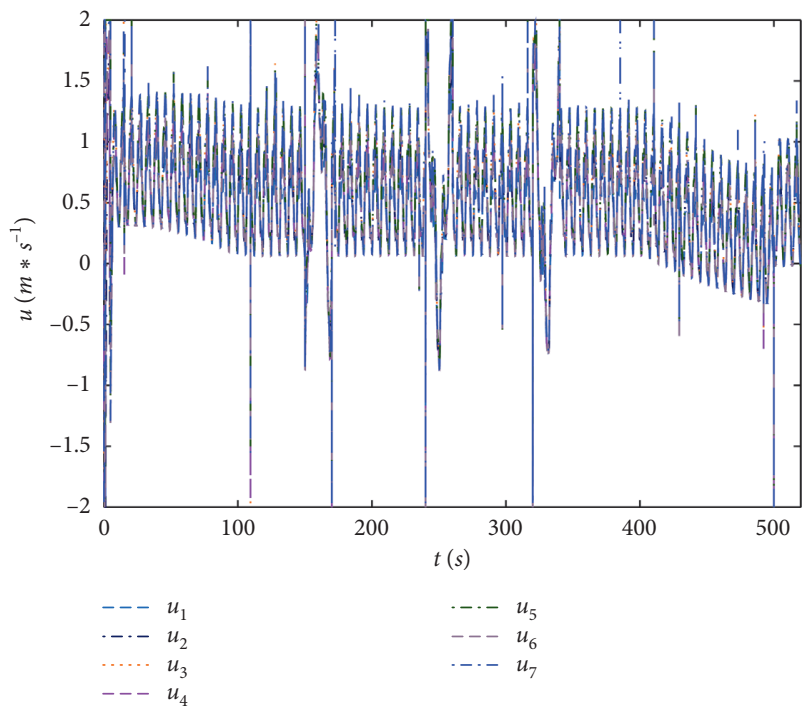

(d)

Figure 4: The simulation results of each vehicle with uncertain driving resistance, actuator saturation, and reduced chattering: (a) the velocity curves; (b) the position curves; (c) the distances curves between two neighbor vehicles; (d) the control input curves.

However, it triggers the chattering phenomenon, which may deteriorate the vehicle actuator and lead to the driving uncomfortableness for passengers.

To avoid chattering in practical implementation, the modified control algorithms (47) and (49) are proposed and simulations are performed in Case 3.

Case 3. Platoon with reduced chattering

In this case, the control laws (47) and (49) and coefficients adaptive laws (48) and (50) are applied to the vehicle platoon. The sigmoid-like function $\left(\delta_{i} /\left(\left|\delta_{i}+\varsigma\right|\right)\right)$ is used to replace the sgn $(\cdot)$, where

$$
\varsigma=1 * 10^{-5} \text {. }
$$

Figure 4(a) shows the vehicle velocities and velocity errors (between followers and leader). Compared with Figure 3(a), the amendment control algorithms (47) and (49) have not greatly influenced the performance of vehicle velocities and velocity errors. From Figures 4(b) and 4(c), one can see that the proposed amendment algorithms (47) and (49) can also maintain a desired intervehicle distance with their neighbors, which ensures the driving safety of vehicle platoon. Figure 4(d) illustrates the control input of all followers; it is obvious that influence of chatting is significantly 
weakened compared with Figure 3(d). Therefore, better vehicle platoon performance can be obtained and the driving comfortableness can be enhanced.

Remark 3. In Case 1, we only consider the influence of uncertain driving resistance for vehicle platoon, where (19), (20), (22), and (23) are applied to the vehicle. However, it exceeds the maximum output of vehicle actuator and causes the phenomena of actuator saturation. Therefore, we consider the problem of actuator saturation in Case 2. And the control laws (34), (35), and (37) and coefficients adaptive laws (36) and (38) are applied to the vehicle platoon. However, it triggers the chattering phenomenon in simulation, which may deteriorate the vehicle actuator and lead to the driving uncomfortableness for passengers. To avoid chattering in practical implementation, the modified control algorithm (47), (49) is proposed and simulations are performed in Case 3. Finally, better vehicle platoon performance is obtained and the driving comfortableness is enhanced.

\section{Conclusions}

In this paper, we discuss the distributed adaptive control problem for vehicle platoon with uncertain driving resistance and actuator saturation. Coupled sliding surface (CSS) is deployed to link interconnected vehicles and an adaptive control method is adopted to estimate the variations of resistance coefficients. An antiwindup compensation based approach is utilized to attenuate the integral windup of the adaptive platoon control laws in case of actuator saturation. Theoretical results are verified via numerical simulations, which demonstrate that the proposed control algorithm can make every vehicle keep the desired distance with the preceding vehicle and all followers' velocity will gradually converge to the velocity of the leader even in the presence of uncertain driving resistance coefficients and actuator saturation.

\section{Data Availability}

The data used to support the findings of this paper are included within the article and are available from the corresponding author upon request.

\section{Disclosure}

Part of the content of this paper has been accepted for a podium presentation at the 36th Chinese Control Conference (CCC).

\section{Conflicts of Interest}

The authors declare that they have no conflicts of interest.

\section{Acknowledgments}

This work was supported by the Fundamental Research Funds for the Central University of China-Excellent Doctoral Dissertation Foundation of Chang'an University (no.
300102320720) and the Natural Science Basic Research Plan in Shaanxi Province of China (no. 2020JM-255).

\section{References}

[1] E. Ahmed and H. Gharavi, "Cooperative vehicular networking: a survey," IEEE Transactions on Intelligent Transportation Systems, vol. 19, no. 3, pp. 996-1014, 2018.

[2] M. Taiebat, A. L. Brown, H. R. Safford, S. Qu, and M. Xu, "A review on energy, environmental, and sustainability implications of connected and automated vehicles," Environmental Science \& Technology, vol. 52, no. 20, pp. 11449-11465, 2018.

[3] S. E. Shladover, "PATH at 20-history and major milestones," IEEE Transactions on Intelligent Transportation Systems, vol. 8, no. 4, pp. 584-592, 2007.

[4] E. Coelingh and S. Solyom, "All aboard the robotic road train," IEEE Spectrum, vol. 49, no. 11, pp. 34-39, 2012.

[5] Z. Wang, Y. Bian, S. E. Shladover, G. Wu, S. E. Li, and M. J. Barth, "A survey on cooperative longitudinal motion control of multiple connected and automated vehicles," IEEE Intelligent Transportation Systems Magazine, vol. 12, no. 1, pp. 4-24, 2020.

[6] M. di Bernardo, P. Falcone, A. Salvi, and S. Santini, "Design, analysis, and experimental validation of a distributed protocol for platooning in the presence of time-varying heterogeneous delays," IEEE Transactions on Control Systems Technology, vol. 24, no. 2, pp. 413-427, 2016.

[7] M. di Bernardo, S. A. Salvi, and S. Santini, "Distributed consensus strategy for platooning of vehicles in the presence of time-varying heterogeneous communication delays," IEEE Transactions on Intelligent Transportation Systems, vol. 16, no. 1, pp. 102-112, 2015.

[8] S. Santini, A. S. A. Salvi, M. SegataPescape, and R. Lo Cigno, "A consensus-based approach for platooning with intervehicular communications and its validation in realistic scenarios," IEEE Transactions on Vehicular Technology, vol. 66, no. 3, pp. 1985-1999, 2017.

[9] J.-W. Kwon and D. Chwa, "Adaptive bidirectional platoon control using a coupled sliding mode control method," IEEE Transactions on Intelligent Transportation Systems, vol. 15, no. 5, pp. 2040-2048, 2014.

[10] Y. Li, K. Li, T. Zheng, X. Hu, H. Feng, and Y. Li, "Evaluating the performance of vehicular platoon control under different network topologies of initial states," Physica A: Statistical Mechanics and Its Applications, vol. 450, pp. 359-368, 2016.

[11] P. Yang, M. Y. Tang, and X. Zhu, "Consensus based control algorithm for nonlinear vehicle platoons in the presence of time delay," International Journal of Control, Automation and Systems, vol. 17, no. 3, pp. 752-764, 2019.

[12] D. Swaroop, S. B. Hedrick, and S. B. Choi, "Direct adaptive longitudinal control of vehicle platoons," IEEE Transactions on Vehicular Technology, vol. 50, no. 1, pp. 150-161, 2001.

[13] M. Yan, J. Song, P. Yang, and Y. Tang, "Distributed adaptive sliding mode control for vehicle platoon with uncertain driving resistance," in Proceedings of the 36th Chinese Control Conference (CCC) 2017, pp. 9396-9400, IEEE, Dalian, China, July 2017.

[14] X. Guo, F. J. Wang, and R. S. H. Teo, "Distributed adaptive integrated-sliding-mode controller synthesis for string stability of vehicle platoons," IEEE Transactions on Intelligent Transportation Systems, vol. 17, no. 9, pp. 2419-2429, 2016.

[15] V. Filaretov, A. Zhirabok, A. Zuev, and A. Procenko, "The development of system of accommodation to faults of navigation sensors of underwater vehicles with resistance to 
disturbance," in Proceedings of the 14th International Conference on Control, Automation and Systems (ICCAS), 2014, pp. 1548-1553, IEEE, Seoul, Republic of Korea, October 2014.

[16] C. E. Tannoury, S. Moussaoui, F. Plestan, N. Romani, and G. Pita-Gil, "Synthesis and application of nonlinear observers for the estimation of tire effective radius and rolling resistance of an automotive vehicle," IEEE Transactions on Control Systems Technology, vol. 21, no. 6, pp. 2408-2416, 2013.

[17] G. Shi-Gen, D. Hai-Rong, N. Bin et al., "Cooperative adaptive bidirectional control of a train platoon for efficient utility and string stability," Chinese Physics B, vol. 24, no. 9, Article ID 090506, 2015.

[18] S. Altmannshofer and C. Endisch, "Robust vehicle mass and driving resistance estimation," in Proceedings of the American Control Conference (ACC), 2016, pp. 6869-6874, IEEE, Boston, MA, USA, July 2016.

[19] M. Chen, S. S. Ge, and B. Ren, "Adaptive tracking control of uncertain MIMO nonlinear systems with input constraints," Automatica, vol. 47, no. 3, pp. 452-465, 2011.

[20] S. Gao, H. Dong, Y. Chen, B. Ning, G. Chen, and X. Yang, "Approximation-based robust adaptive automatic train control: an approach for actuator saturation," IEEE Transactions on Intelligent Transportation Systems, vol. 14, no. 4, pp. 1733-1742, 2013.

[21] Y. Li, S. Tong, and T. Li, "Adaptive fuzzy output-feedback control for output constrained nonlinear systems in the presence of input saturation," Fuzzy Sets and Systems, vol. 248, pp. 138-155, 2014.

[22] W. Sun, Z. Zhao, and H. Gao, "Saturated adaptive robust control for active suspension systems," IEEE Transactions on Industrial Electronics, vol. 60, no. 9, pp. 3889-3896, 2013.

[23] S. Li, L. Yang, and Z. Gao, "Adaptive coordinated control of multiple high-speed trains with input saturation," Nonlinear Dynamics, vol. 4, no. 83, pp. 2157-2169, 2016.

[24] M. Yan, Y. Tang, P. Yang, and L. Zuo, "Consensus based platoon algorithm for velocity-measurement-absent vehicles with actuator saturation," Journal of Advanced Transportation, vol. 2017, Article ID 8023018, 8 pages, 2017.

[25] R. Rajamani, Vehicle Dynamics and Control, Springer Science \& Business Media, Berlin, Germany, 2011.

[26] Y. Cao, D. Stuart, W. Ren, and Z. Meng, "Distributed containment control for multiple autonomous vehicles with double-integrator dynamics: algorithms and experiments," IEEE Transactions on Control Systems Technology, vol. 19, no. 4, pp. 929-938, 2011.

[27] H. Nijmeijer and A. Van Der Schaft, Nonlinear Dynamical Control Systems, Springer, Berlin, Germany, 2013. 